\title{
Differential expression of multidrug resistance-related proteins in adriamycin-resistant (pumc-91/ADM) and parental (pumc-91) human bladder cancer cell lines
}

\author{
MAN ZHAO $^{1 *}$, SHULIANG YU ${ }^{1 *}$ and MAN ZHANG ${ }^{1,2}$ \\ ${ }^{1}$ Clinical Laboratory Medicine and ${ }^{2}$ Beijing Key Laboratory of Urinary Cellular Molecular Diagnostics, \\ Beijing Shijitan Hospital, Capital Medical University, Beijing 100038, P.R. China
}

Received July 27, 2015; Accepted August 16, 2016

DOI: $10.3892 / \mathrm{mmr} .2016 .5806$

\begin{abstract}
Multidrug resistance (MDR) is the major obstacle to bladder cancer chemotherapy. Several mechanisms have been implicated in the development of MDR, including extrusion of the drug by cell membrane pumps, associated with P-glycoprotein (P-gp) and multidrug resistance-associated protein (MRP); increased DNA damage repair, associated with topoisomerase II (Topo II); suppression of drug-induced apoptosis, associated with p53; and regulation of cancer cell growth, associated with vascular endothelial growth factor (VEGF). In the present study, the expression levels of these five markers were detected in an adriamycin (ADM)-resistant human bladder cancer cell line (pumc-91/ADM) and its parental cell line (pumc-91), in order to determine which marker is more important, or whether all of them participate in drug resistance. The expression levels of P-gp, MRP, Topo II, VEGF and p53 were measured in the two cell lines by reverse transcription-quantitative polymerase chain reaction, western blotting and immunohistochemistry. A significant increase in P-gp, MRP and VEGF, and a decrease in Topo II mRNA expression were detected in the pumc-91/ADM drug-resistant cell line compared with the pumc-91 cell line; however, no difference in p53 mRNA expression was detected between the cells. In pumc-91/ADM cells, the protein expression levels of P-gp and MRP were upregulated, whereas Topo II was significantly decreased. However, no marked differences in p53 or VEGF expression were detected between the two cell lines at the protein level. The cytoplasmic and cell membrane localization
\end{abstract}

Correspondence to: Dr Man Zhang, Clinical Laboratory Medicine, Beijing Shijitan Hospital, Capital Medical University, 10 Tieyi Road, Haidian, Beijing 100038, P.R. China

E-mail: mzhang99@aliyun.com

*Contributed equally

Key words: bladder cancer, P-glycoprotein, topoisomerase II, p53, multidrug resistance-associated protein, vascular endothelial growth factor, multidrug resistance of P-gp and MRP, the cytoplasmic localization of VEGF, and the nuclear localization of p53 and Topo II were confirmed in the two cell lines. The present study detected increased P-gp and MRP, and reduced Topo II expression in pumc-91/ADM cells compared with pumc-91 cells; however, no difference was detected in p53 and VEGF expression between the cell lines. In conclusion, a significant upregulation of MRP and downregulation of Topo II were detected in the ADM-resistant human bladder cancer cell line (pumc-91/ADM) compared with in the parental cell line (pumc-91).

\section{Introduction}

Bladder cancer, which is the second most common malignant tumor of the urinary system, is ranked as the fourth most common cancer in males and the eighth leading cause of cancer-associated deaths worldwide (1). Intravesical administration of chemotherapy drugs and adjuvant chemotherapy after surgery have been the standard treatment of bladder cancer for $>20$ years (2). The five-year survival rate of patients with bladder cancer is $72.8 \%$ in men and $69.3 \%$ in women (3). Although chemotherapeutic drugs, including doxorubicin, vincristine, cisplatin and methotrexate, are widely used in the radical and palliative treatment of human bladder cancer, multidrug resistance (MDR) is one of the major drawbacks of bladder cancer chemotherapy. MDR is a significant obstacle to successful cancer treatment, which contributes to $>90 \%$ of all treatment failures in patients with bladder cancer (4). Therefore, further studies regarding MDR in bladder cancer are urgently required.

MDR is a complex process associated with numerous factors in various types of cancer. In several instances, resistance is achieved by an increased efflux of chemotherapeutic agents out of the tumor. For example, P-glycoprotein (P-gp) and multidrug resistance-associated protein (MRP) are two classical efflux proteins, which result in drug resistance by pumping drugs out of the cell. Alterations to the drug target enzyme activity are also associated with drug resistance. Dysregulation of topoisomerase II (Topo II) results in the dissociation of cleavable complexes and reduction of DNA damage, which consequently induces drug resistance. Apoptotic cell death is the most common underlying 
mechanism of antineoplastic drugs; however, the excessive expression of anti-apoptotic genes, such as p53, may lead to chemotherapy resistance. Microenvironmental resistance is another important factor; changes to the tumor microenvironment due to the action of vascular endothelial growth factor (VEGF) may also result in MDR (5-9).

Our laboratory has successfully established an adriamycin (ADM)-resistant human bladder cancer cell line (pumc-91/ADM) from the parental cell line pumc-91. According to drug resistance spectrum analysis, the pumc-91/ADM cell line exhibited the characteristics of MDR (10). In the present study, the expression levels of the five markers were analyzed in pumc-91/ADM and pumc-91 cells in order to determine the relationship between protein expression and drug resistance. In addition, the possible pathways that may lead to bladder cancer MDR were evaluated. The results may provide the basis for the knockdown or transfection of a target to reverse MDR in the future.

\section{Materials and methods}

Cell culture. The pumc-91 human bladder cancer cell line was generously provided by the Peking Union Medical College Hospital(Beijing,China). The increasing concentration gradient method was adopted in vitro to induce resistance. Increasing ADM concentrations $(0.3,0.6$ and $1.0 \mu \mathrm{g} / \mathrm{ml})$ were used to establish pumc-91/ADM multidrug-resistant bladder cell lines. The half maximal inhibitory concentration $\left(\mathrm{IC}_{50}\right)$ values for ADM treatment in pumc-91 and pumc-91/ADM cells were 0.61 and $6.02 \mu \mathrm{M}$, respectively. The $\mathrm{IC}_{50}$ for pumc-91/ADM was 9.86-fold higher than that of pumc-91, thus indicating that the ADM-resistant cell line pumc-91/ADM $(1.0 \mu \mathrm{g} / \mathrm{ml})$ was successfully established. Pumc-91 and pumc-91/ADM cells were cultured in RPMI 1640 medium (Gibco; Thermo Fisher Scientific, Inc., Waltham, MA, USA) supplemented with 10 and $18 \%$ fetal bovine serum (Beijing Dingguo Biotechnology Co., Ltd., Beijing, China), respectively. Cells were maintained at $37^{\circ} \mathrm{C}$ in an atmosphere containing $5 \% \mathrm{CO}_{2}$.

Drug cytotoxicity analysis. To analyze drug cytotoxicity, $2.0 \times 10^{4}$ cells/well were cultured with a concentration gradient $(0.3,0.6$ and $1.0 \mu \mathrm{g} / \mathrm{ml})$ of ADM (Sigma-Aldrich; Merck Millipore, Darmstadt, Germany) in 96 -well plates at $37^{\circ} \mathrm{C}$. Following $72 \mathrm{~h}$ of drug treatment, $20 \mu 1$ 3-(4,5-dimethylthiazol-2-yl)-2,5-diphenyltetrazolium bromide was added to the cells. Following a $4 \mathrm{~h}$ incubation at $37^{\circ} \mathrm{C}$, absorbance was measured in the presence of dimethyl sulfoxide at $450 \mathrm{~nm}$. Cell survival in the absence of the drug was defined as $100 \%$ cell survival. The $\mathrm{IC}_{50}$ values of $\mathrm{ADM}$ were determined from the corresponding absorbance.

Reverse transcription-quantitative polymerase chain reaction (RT-qPCR). Total RNA was isolated from the cells using TRIzol $^{\circledR}$ reagent (Invitrogen; Thermo Fisher Scientific, Inc.) as previously described (11). Total RNA ( $2 \mu \mathrm{g})$ was then reverse transcribed to cDNA using the Reverse Transcription system according to the manufacturer's protocol (Promega Corporation, Madison, WI, USA). TransStart Top Green qPCR SuperMix (Beijing TransGen Biotech Co., Ltd., Beijing, China) and Roche LightCycler 480 Real Time PCR system (Applied
Biosystems; Thermo Fisher Scientific, Inc.) were used to conduct RT-qPCR, according to the manufacturers' protocols. The PCR conditions were as follows: 45 cycles of annealing at $55^{\circ} \mathrm{C}$ for $1 \mathrm{~min}$, denaturation at $95^{\circ} \mathrm{C}$ for $40 \mathrm{sec}$, and elongation at $72^{\circ} \mathrm{C}$ for $1 \mathrm{~min}$. The PCR primer sequences are presented in Table I. The $2^{-\Delta \Delta C q}$ method was applied for relative quantitative analysis (12). All samples were independently analyzed at least three times in duplicate.

Western blot analysis. Pumc-91 and pumc-91/ADM cells were grown to $80-90 \%$ confluence. Cells were harvested, washed three times with phosphate-buffered saline (PBS), and lysed with radioimmunoprecipitation assay cell lysis buffer (Beijing Dingguo Biotechnology Co., Ltd.). The protein was quantified using Bicinchoninic Acid kit (Sigma-Aldrich; Merck Millipore) and protein samples $(60 \mu \mathrm{g})$ were loaded onto an $8 \%$ sodium dodecyl sulfate-polyacrylamide gel and were electrotransferred to polyvinylidene fluoride membranes. After blocking with 5\% nonfat dry milk for $2 \mathrm{~h}$ at room temperature, the membranes were incubated with the following primary antibodies: P-gp (1:2,000; cat. no. ab170903; Abcam, Cambridge, MA, USA), Topo II (1:10,000; cat. no. ab12318; Abcam), MRP (1:500; cat. no. sc-18835; Santa Cruz Biotechnology, Inc., Dallas, TX, USA), p53 (1:500; cat. no. sc-126; Santa Cruz Biotechnology, Inc.), VEGF (1:500; cat. no. sc-65617; Santa Cruz Biotechnology, Inc.) and $\beta$-actin (1:400; cat. no. TA-09; Beijing Zhongshan Jinqiao Biotechnology Co., Ltd., Beijing, China) at $4^{\circ} \mathrm{C}$ overnight. Subsequently, the membranes were incubated with goat anti-mouse immunoglobulin (Ig) G (1:1,000; Beijing Dingguo Biotechnology Co., Ltd.; cat. no. IH-0031) or goat anti-rabbit IgG (1:1,000; Beijing Dingguo Biotechnology Co., Ltd.; cat. no. IH-0011) for $30 \mathrm{~min}$ at $37^{\circ} \mathrm{C}$. After extensive washing with PBS with Tween 20, proteins were visualized using an enhanced horseradish peroxidase-3,3'-diaminobenzidine (DAB) chromogenic kit (Tiangen Biotech Co., Ltd., Beijing, China), according to the manufacturer's protocol. Lane 1D gel analysis software (Beijing Sage Creation Science Co., Ltd., Beijing, China) was used to analyze the bands.

Immunohistochemistry. For staining, the pumc-91 and pumc-91/ADM cell slides were fixed with $4 \%$ paraformaldehyde for $15 \mathrm{~min}$ at room temperature. After washing with PBS, the slides were treated with $50 \mu 1$ hydrogen peroxide (Beijing Dingguo Biotechnology Co., Ltd.) for $20 \mathrm{~min}$ at room temperature to block the action of endogenous peroxidases. Subsequently, the slides were blocked in nonimmune serum (Beijing Dingguo Biotechnology, Co., Ltd.) at $37^{\circ} \mathrm{C}$ for $30 \mathrm{~min}$. The specimens were then incubated with the following primary antibodies: P-gp (1:400), Topo II (1:10,000), MRP (1:400), p53 (1:500), VEGF (1:500) and $\beta$-actin $(1: 200)$ at $37^{\circ} \mathrm{C}$ for $1 \mathrm{~h}$, followed by incubation with secondary antibodies $(1: 1,000$; EnVision Detection systems, Peroxidase/DAB, Rabbit/Mouse; Dako, Carpinteria, CA, USA) for $30 \mathrm{~min}$ at $37^{\circ} \mathrm{C}$. A DAB kit was used to visualize the immunohistochemical staining, and slides were counterstained with hematoxylin. Finally, specimens were dehydrated in gradient alcohol. The slides were observed using an Olympus CX31 microscope (Olympus Corporation, Tokyo, Japan) and the staining intensity and percentage of stained cells were quantified according to a 
Table I. Primers for reverse transcription-quantitative polymerase chain reaction.

\begin{tabular}{lll}
\hline Gene & Forward $(5 ' \rightarrow 3$ ') & Reverse $\left(5^{\prime} \rightarrow 3^{\prime}\right)$ \\
\hline P-gp & 5'-GATATTGCCTGGTTTGATGA-3' & 5'-TGCATTTTGTGTTAAGACGC-3' \\
MRP & 5'-TTGCCGTCTACGTGACCATT-3' & 5'-AGGCGTTTGAGGGAGACACT-3' \\
Topo II & 5'-AGGCATCGCATCTTGTTTAG-3' & 5'-CTGTCTCCGGTCTTCCATAA-3' \\
p53 & 5'-CACTAAGCGAGCACTGTCCA-3' & 5'-TTCAGCTCTCGGAACATCTC-3' \\
VEGF & 5'-CAATCGAGACCCTGGTGGACA-3' & 5'-TGTTGGACTCCTCAGTGGGCA-3' \\
GAPDH & 5'-TTTGGTATCGTGGAAGGACT-3' & 5'-AGTAGAGGCAGGGATGATGT-3'
\end{tabular}

P-gp, P-glycoprotein; MRP, multidrug resistance-associated protein; Topo II, topoisomerase II; VEGF, vascular endothelial growth factor; GAPDH, glyceraldehyde 3-phosphate dehydrogenase.

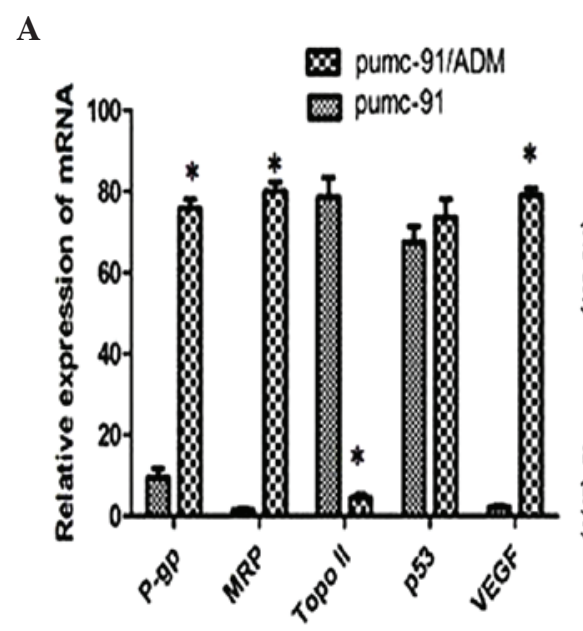

B

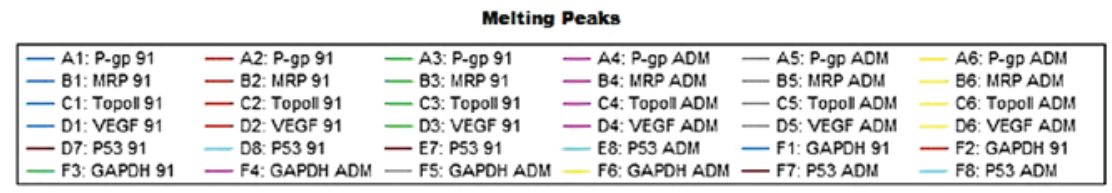

Figure 1. (A) mRNA expression levels of P-glycoprotein (P-gp), multidrug resistance-associated protein (MRP), topoisomerase II (Topo II), p53 and vascular endothelial growth factor (VEGF) in pumc-91 and adriamycin (ADM)-resistant pumc-91/ADM cells. (B) Melting curve exhibited unimodal distribution, thus indicating that the amplification products exhibited the characteristics of specificity. $2^{-\Delta \triangle C q}$ method was applied for relative quantitative analysis. The experiments were repeated at least three times. Data are presented as the mean \pm standard deviation. ${ }^{*} \mathrm{P}<0.05$ vs. pumc-91 cells. GAPDH, glyceraldehyde 3-phosphate dehydrogenase.

previously described approach (13). Professional image analysis software, Image Pro Plus 6.0 (Media Cybernetics, Inc., Rockville, MD USA) was used to evaluate the results.

Statistical analysis. All data are presented as the mean \pm standard deviation of at least three independent experiments. The differences between groups were assessed by Student's t-test. Statistical analysis was carried out using SPSS software version 16.0 (SPSS Inc., Chicago, IL, USA). P<0.05 was considered to indicate a statistically significant difference.

\section{Results}

Differential expression of $m R N A$ in the two cell lines. The mRNA expression levels of P-gp, MRP, Topo II, p53 and VEGF were detected by RT-qPCR. Glyceraldehyde 3-phosphate dehydrogenase was used as an endogenous control. The mRNA expression levels of P-gp, MRP and VEGF were markedly upregulated, whereas Topo II was downregulated in the pumc-91/ADM cells compared with the pumc-91 cell line $(\mathrm{P}=0.017,0.045,0.029$ and 0.004, respectively; Fig. 1). These differences were statistically significant. However, no difference was detected in p53 expression at the genetic level between the two cell lines ( $\mathrm{P}=0.722$; Fig. 1).

Differential expression of proteins in the two cell lines. The corresponding protein expression levels were confirmed by western blot analysis in the two cell lines. Compared with pumc-91 cells, a significant increase in P-gp and MRP, and decrease in Topo II was detected in the pumc-91/ADM drug-resistant cell line $(\mathrm{P}=0.008,0.031$ and 0.014 , respectively; Fig. 2). These differences were statistically significant. However, no evident differences in p53 and VEGF expression were detected between the two cell lines at the protein level ( $\mathrm{P}=0.103$ and 0.700 , respectively; Fig. 2).

Immunohistochemical protein expression in the two cell lines. The results of the expression analyses were further confirmed by localization of P-gp, MRP, Topo II, p53 and VEGF by immunohistochemistry in the two cell lines. Image Pro Plus 6.0 was used to evaluate the results. $\beta$-actin was used as a positive control, whereas PBS was used instead of the primary antibodies as a negative control. The cytoplasmic and cell membrane localization of P-gp and MRP, 
A
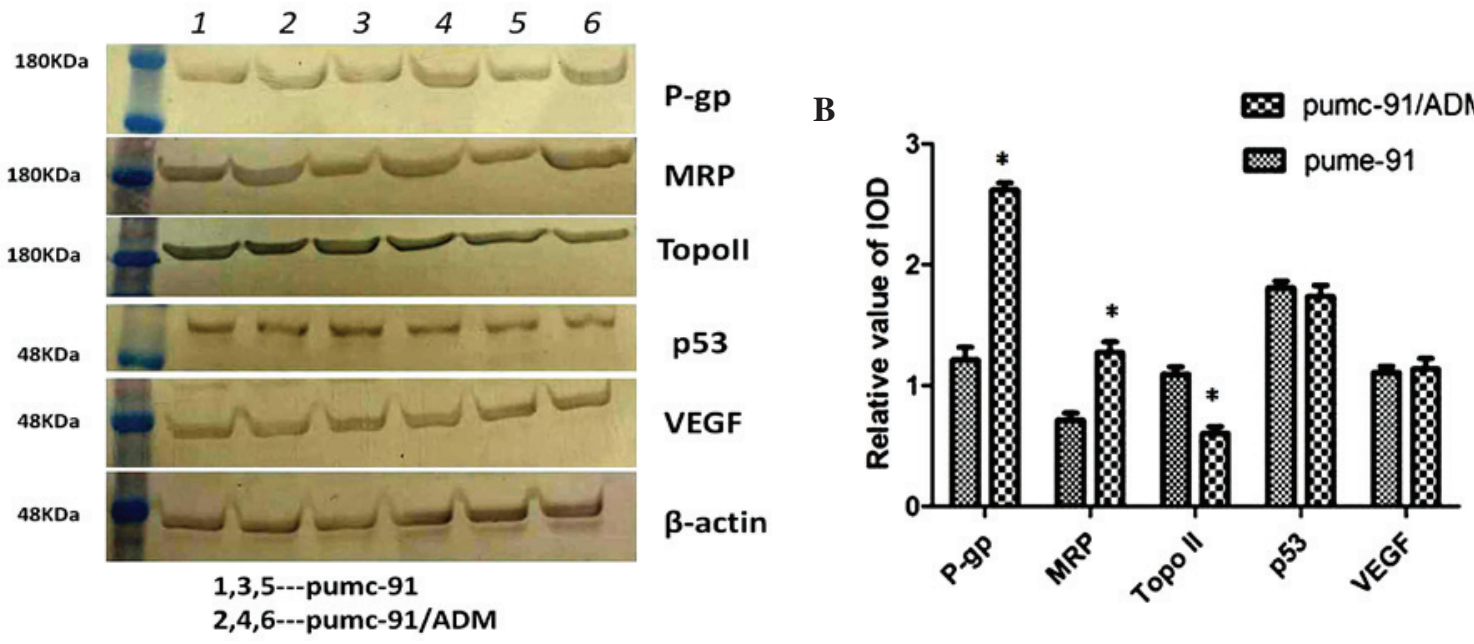

Figure 2. (A) P-glycoprotein (P-gp), multidrug resistance-associated protein (MRP), topoisomerase II (Topo II), p53 and vascular endothelial growth factor (VEGF) protein expression levels were verified by western blotting. $\beta$-actin was used as an internal control. (B) Integrated optical density (IOD) value of each band was analyzed using Lane 1D gel analysis software. All samples were independently analyzed three times in duplicate. Data are presented as the mean \pm standard deviation. ${ }^{*} \mathrm{P}<0.05$ vs. pumc- 91 cells. ADM, adriamycin.
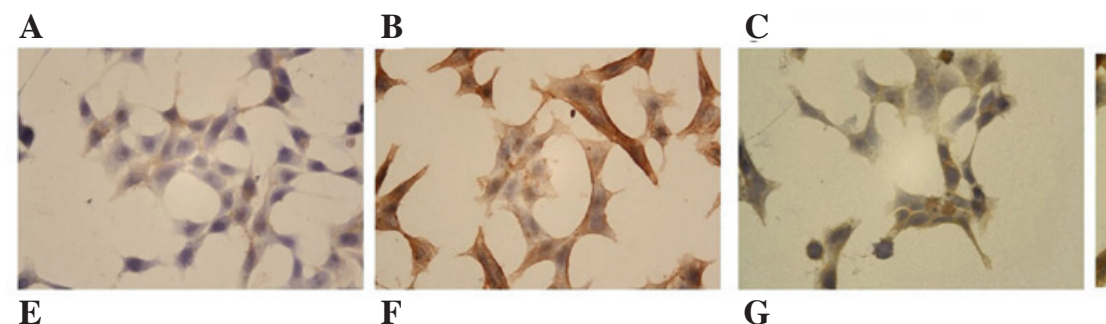

D
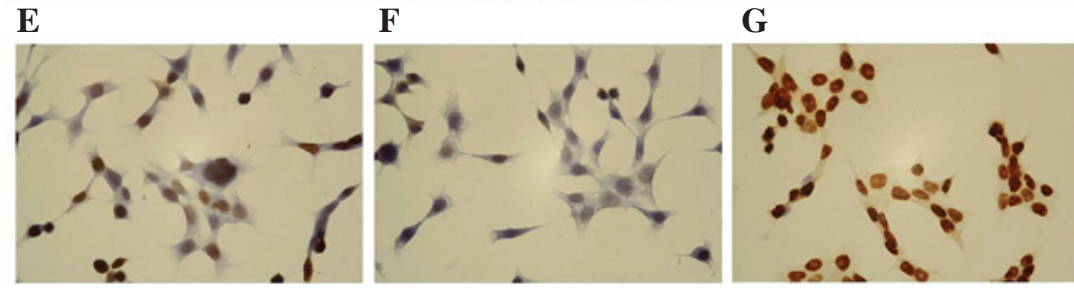

I

$\mathbf{J}$

\section{$\mathbf{K}$}

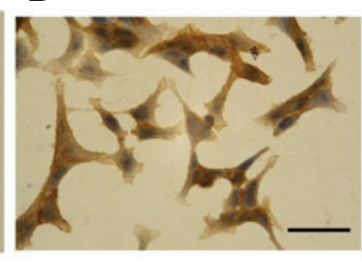

$\mathbf{H}$
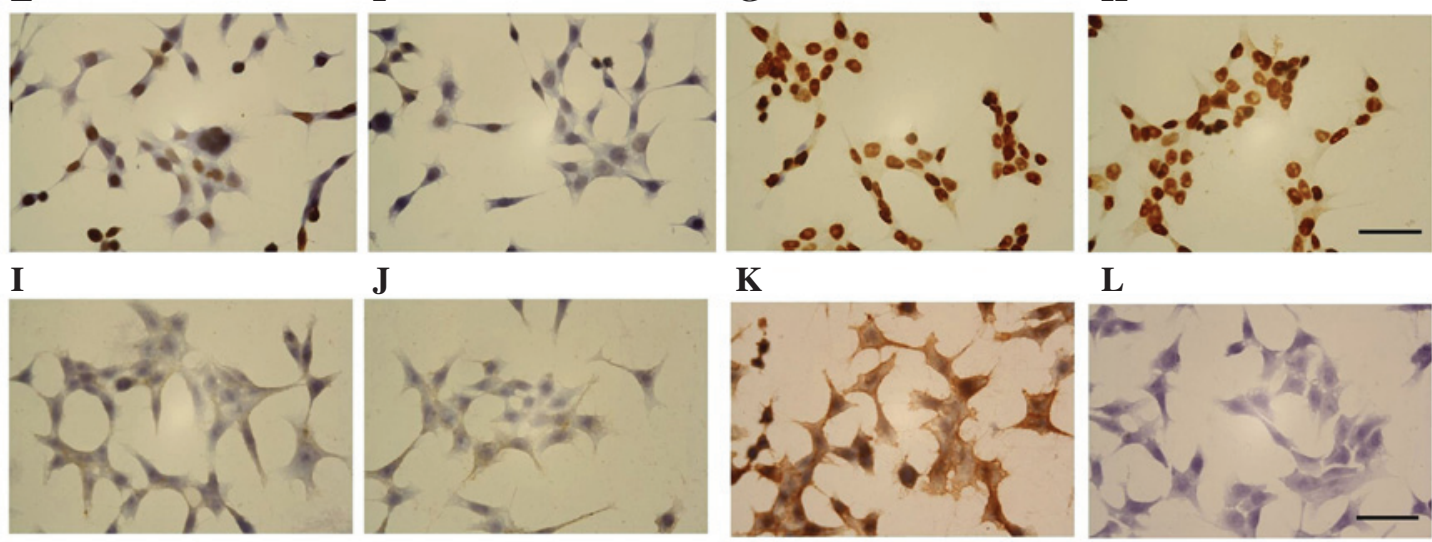

$\mathbf{L}$

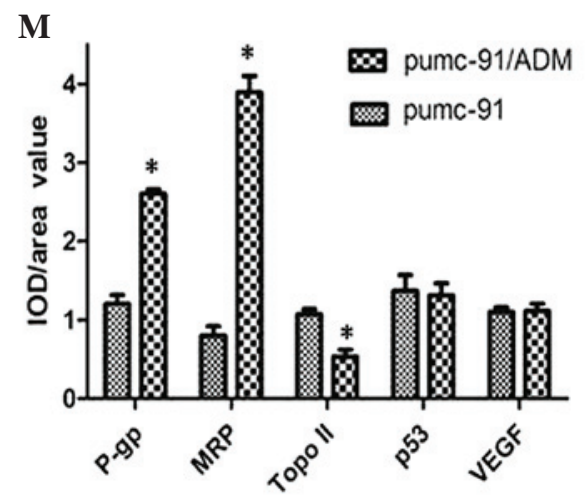

Figure 3. Immunohistochemical staining of P-glycoprotein (P-gp), multidrug resistance-associated protein (MRP), topoisomerase II (Topo II), p53 and vascular endothelial growth factor (VEGF) expression in pumc-91 and adriamycin (ADM)-resistant pumc-91/ADM cell lines. P-gp in (A) pumc-91 and (B) pumc-91/ADM cells. MRP in (C) pumc-91 and (D) pumc-91/ADM cells. Topo II in (E) pumc-91 and (F) pumc-91/ADM cells. p53 in (G) pumc-91 and (H) pumc-91/ADM cells. VEGF in (I) pumc-91 and (J) pumc-91/ADM cells. (K) $\beta$-actin was used as a positive control. (L) Phosphate-buffered saline instead of primary antibodies was used as a negative control. (M) Protein expression was presented as integrated optical density (IOD)/area of at least three independent experiments. Data are presented as the mean \pm standard deviation. " $\mathrm{P}<0.05$ vs. pumc-91 cells. Magnification, $\mathrm{x} 100$. 
the cytoplasmic localization of VEGF, and the nuclear localization of p53 and Topo II were observed in the two cell lines. Consistent with the results of western blotting, P-gp and MRP were upregulated, and Topo II was downregulated in pumc-91/ADM cells compared with in pumc-91 cells $(\mathrm{P}=0.014,0.048$ and 0.039 , respectively; Fig. 3). No significant difference in p53 and VEGF expression was detected between the two cell lines $(\mathrm{P}=0.316$ and 0.113 , respectively; Fig. 3).

\section{Discussion}

Adjuvant chemotherapy following surgery is considered necessary for the treatment of bladder cancer; however, long-term chemotherapy decreases the response of cancer cells to anticancer agents, thus leading to the occurrence of tumor escape. In the majority of cancer types, several key molecules participate in the development of MDR, including P-gp, MRP, Topo II, p53 and VEGF (14-19). The present study investigated the relationship between the expression levels of these five markers and drug resistance in ADM-resistant (pumc-91/ADM) and parental (pumc-91) human bladder cancer cell lines. The results provided evidence regarding the possible pathways that lead to bladder cancer MDR. In addition, the results may provide useful information for the reversal of MDR in bladder cancer.

Among the five molecules evaluated in the present study, aberrant expression of P-gp and MRP has been the most extensively reported. P-gp and MRP are two important members of the ATP-binding cassette transporter superfamily, which have been reported to confer resistance against various chemotherapeutic agents (20). By affecting the intracellular drug concentration through drug efflux alterations, P-gp and MRP are associated with drug resistance in various types of cancer. The results of the present study suggested that P-gp and MRP were upregulated in pumc-91/ADM cells compared with in pumc-91 cells. Notably, MRP demonstrated a more significant increasing trend. The mechanisms underlying the effects of MRP on MDR include effects on drug efflux and microenvironment alterations. MRP is able to redistribute intracellular drugs, via altering the $\mathrm{pH}$ microenvironment of the cytoplasm and organelles, which may trigger resistance (21). Therefore, in further studies regarding the reversal of MDR, researchers have selected MRP as the first factor to target by transfection or expression knockdown. Copsel et al reported that knockdown of MRP by short hairpin RNA could regulate drug resistance in the U937 acute myeloid leukemia cell line (22). In addition, $\mathrm{Su}$ and Pasternak revealed that downregulation of MRP significantly enhanced the analgesic potency of systemic morphine in MRP knockout mice (23).

Topo II is a nuclear protein that is usually highly expressed during active cell proliferation; therefore, overexpression of Topo II is common in malignant tumors. However, it is generally believed that decreased expression of Topo II is associated with tumor drug resistance. Yu et al investigated the expression of Topo II in gastric cancer following chemotherapy using immunohistochemistry; Topo II staining was negative whereas other MDR-related proteins exhibited positive staining (24). Tsang et al hypothesized that drug resistance could be induced by downregulation of Topo II in
A431 human squamous carcinoma cells (25). Notably, in the present study, Topo II was downregulated in pumc-91/ADM cells compared with in pumc-91 cells. The detailed mechanism underlying the participation of Topo II downregulation in bladder cancer MDR remains to be elucidated. Notably, Topo II has a role in formation of DNA complexes in the $\mathrm{S}-\mathrm{G}_{2}-\mathrm{M}$ phase of the cell cycle. Understanding the mode of action of Topo II in MDR in other types of cancer may provide certain possibilities for its underlying mechanism in bladder cancer MDR. Topo II has been previously reported to be significantly decreased in gastric cancer cells resistant to ADM and mitomycin (26). The downregulation of Topo II may alter the crosslinking and production of DNA complexes, resulting in a decline in chemosensitivity. In addition, treatment with a Topo II inhibitor resulted in MDR in lung cancer cells via the epidermal growth factor receptor signaling pathway (27).

P53 has an important role in cell cycle regulation and apoptosis induction in bladder cancer. Chin and Kong reported that p53 markedly reduced the sensitivity of tumor cells towards chemotherapy by activating the initiator of MDR-1, inducing the duplication of MDR-1 (28). Garufi and D'Orazi demonstrated that inactivation of $\mathrm{p} 53$ reduced the tumor cell response to drugs, thus inducing drug resistance (29). However, discordance exists between the results of the present study and the aforementioned reports with regards to the expression of p53. In the present study, the expression levels of p53 did not differ between the drug-resistant pumc-91/ADM cell line and the pumc-91 cell line.

There have been few reports regarding the relationship between VEGF and drug resistance in tumors. Lin et al decreased the mRNA and protein expression of the critical target gene VEGF-A and VEGFR2 in hepatic cancer xenograft tumor tissues (30). In vitro studies conducted by Mésange et al revealed that bevacizumab-resistant cells displayed intrinsically higher VEGF signaling intensity and tolerance compared with their bevacizumab-sensitive counterparts in colorectal cancer (31). In the present study, differential expression of VEGF was detected between the protein and gene level. It may be hypothesized that methylation of cytosine induced the abnormal gene expression, whereas the DNA sequence and gene product remained normal (32). Besides methylation, acetylation may regulate gene transcription, leading to differential expression between the protein and gene (33).

In conclusion, the present study detected a significant upregulation of MRP and downregulation of Topo II in ADM-resistant human bladder cancer cells (pumc-91/ADM) compared with in the parental cells (pumc-91). Further studies are required to explore the specific mechanisms underlying MDR in bladder cancer. In addition, how to reverse MDR based on the targets examined in the present study will be our future aim.

\section{Acknowledgements}

The present study was supported by the Beijing Administration of Traditional Chinese Medical (grant no. 2014-ZYJ04) and Beijing Key Laboratory of Urinary Cellular Molecular Diagnostics (grant no. Z151100001615060). 


\section{References}

1. Lei T, Zhao X, Jin S, Meng Q, Zhou H and Zhang M: Discovery of potential bladder cancer biomarkers by comparative urine proteomics and analysis. Clin Genitourin Cancer 11: 56-62, 2013

2. Meng Q, Lei T, Zhang M, Zhao J, Zhao XH and Zhang M: Identification of proteins differentially expressed in adriamycin-resistant (pumc-91/ADM) and parental (pumc-91) human bladder cancer cell lines by proteome analysis. J Cancer Res Clin Oncol 139: 509-519, 2013.

3. Sacerdote C, Guarrera S, Ricceri F, Pardini B, Polidoro S, Allione A, Critelli R, Russo A, Andrew AS, Ye Y, et al: Polymorphisms in the XRCC1 gene modify survival of bladder cancer patients treated with chemotherapy. Int J Cancer 133: 2004-2009, 2013.

4. Zeekpudsa P, Kukongviriyapan V, Senggunprai L, Sripa B and Prawan A: Suppression of NAD $(\mathrm{P}) \mathrm{H}$-quinone oxidoreductase 1 enhanced the susceptibility of cholangiocarcinoma cells to chemotherapeutic agents. J Exp Clin Cancer Res 33: 11, 2014.

5. Zandvliet M, Teske E and Schrickx JA: Multi-drug resistance in a canine lymphoid cell line due to increased P-glycoprotein expression, a potential model for drug-resistant canine lymphoma. Toxicol In Vitro 28: 1498-1506, 2014.

6. Li W and Song M: Expression of multidrug resistance proteins in invasive ductal carcinoma of the breast. Oncol Lett 8: 2103-2109, 2014.

7. Semiglazova TIu, Klimenko VV, Filatova LV, Chubenko VA, Krivorot'ko PV, Ivanov VG, Turkevich EA, Ivantsov AO, Novikov SN, Semiglazov VV, et al: Markers of effectiveness of preoperative taxane-based chemotherapy for locally advanced breast cancer. Vopr Onkol 59: 363-367, 2013 (In Russian).

8. Grosset AA, Labrie M, Gagné D, Vladoiu MC, Gaboury L, Doucet N and St-Pierre Y: Cytosolic galectin-7 impairs p53 functions and induces chemoresistance in breast cancer cells BMC Cancer 14: 801, 2014.

9. Ki CS, Lin TY, Korc M and Lin CC: Thiol-ene hydrogels as desmoplasia-mimetic matrices for modeling pancreatic cancer cell growth, invasion, and drug resistance. Biomaterials 35: 9668-9677, 2014.

10. Zhang M, Jin S and Zhang M: Establishing human multi-drug resistant bladder cancer cell lines Pumc-91/ADM and evaluating its biological characteristics. J Med Res 38: 70-72, 2009.

11. Yu S, Meng Q, Hu H and Zhang M: Correlation of ANXA1 expression with drug resistance and relapse in bladder cancer. Int J Clin Exp Pathol 7: 5538-5548, 2014.

12. Livak KJ and Schmittgen TD: Analysis of relative gene expression data using real-time quantitative PCR and the 2(-Delta Delta C(T)) Method. Methods 25: 402-408, 2001

13. Hu H, Meng Q, Lei T and Zhang M: Nucleophosmin1 associated with drug resistance and recurrence of bladder cancer. Clin Exp Med 15: 361-369, 2015.

14. Pappas JJ, Petropoulos S, Suderman M, Iqbal M, Moisiadis V, Turecki G, Matthews SG and Szyf M: The multidrug resistance 1 gene Abcb1 in brain and placenta: Comparative analysis in human and guinea pig. PLoS One 9: e111135, 2014.

15. Yu P, Du Y, Cheng X, Yu Q, Huang L and Dong R: Expression of multidrug resistance-associated proteins and their relation to postoperative individualized chemotherapy in gastric cancer. World J Surg Oncol 12: 307, 2014.

16. Kodiakov DS, Klimachëv VV, Avdalian AM, Bobrov IP, Lazarev AF, Lushnikova EL and Nepomiashchikh LM: Topoisomerase IIa expression in correlation with clinical and morphological parameters and proliferation (based on argyrophilic proteins of nucleolar organizer regions and Ki-67 antigen) in lung adenocarcinoma. Vopr Onkol 60: 63-68, 2014 (In Russian).

17. Llovet JM: Focal gains of VEGFA: Candidate predictors of sorafenib response in hepatocellular carcinoma. Cancer Cell 25 $560-562,2014$.
18. Tonigold M, Rossmann A, Meinold M, Bette M, Märken M, Henkenius K, Bretz AC, Giel G, Cai C, Rodepeter FR, et al: A cisplatin-resistant head and neck cancer cell line with cytoplasmic p53(mut) exhibits ATP-binding cassette transporter upregulation and high glutathione levels. J Cancer Res Clin Oncol 140: 1689-1704, 2014.

19. An J, Wang X, Guo P, Zhong Y, Zhang X and Yu Z: Hexabromocyclododecane and polychlorinated biphenyls increase resistance of hepatocellular carcinoma cells to cisplatin through the phosphatidylinositol 3-kinase/protein kinase B pathway. Toxicol Lett 229: 265-272, 2014.

20. Kovalev AA, Tsvetaeva DA and Grudinskaja TV: Role of ABC-cassette transporters (MDR1, MRP1, BCRP) in the development of primary and acquired multiple drug resistance in patients with early and metastatic breast cancer. Exp Oncol 35: 287-290, 2013.

21. Lee YK, Lin TH, Chang CF and Lo YL: Galectin-3 silencing inhibits epirubicin-induced ATP binding cassette transporters and activates the mitochondrial apoptosis pathway via $\beta$-catenin/GSK- $3 \beta$ modulation in colorectal carcinoma. PLoS One 8: e82478, 2013.

22. Copsel S, Garcia C, Diez F, Vermeulem M, Baldi A, Bianciotti LG, Russel FG, Shayo C and Davio C: Multidrug resistance protein 4 (MRP4/ABCC4) regulates cAMP cellular levels and controls human leukemia cell proliferation and differentiation. J Biol Chem 286: 6979-6988, 2011.

23. Su W and Pasternak GW: The role of multidrug resistance-associated protein in the blood-brain barrier and opioid analgesia. Synapse 67: 609-619, 2013.

24. Yu P, Du Y, Cheng X, Yu Q, Huang L and Dong R: Expression of multidrug resistance-associated proteins and their relation to postoperative individualized chemotherapy in gastric cancer. World J Surg Oncol 12: 307, 2014.

25. Tsang WP, Kong SK and Kwok TT: Epidermal growth factor induction of resistance to topoisomerase II toxins in human squamous carcinoma A431 cells. Oncol Rep 16: 789-793, 2006.

26. Campiglio M, Somenzi G, Olgiati C, Beretta G, Balsari A, Zaffaroni N, Valagussa $\mathrm{P}$ and Ménard S: Role of proliferation in HER2 status predicted response to doxorubicin. Int J Cancer 105: 568-573, 2003 .

27. Fillmore CM, Xu C, Desai PT, Berry JM, Rowbotham SP Lin YJ, Zhang H, Marquez VE, Hammerman PS, Wong KK and Kim CF: EZH2 inhibition sensitizes BRG1 and EGFR mutant lung tumours to TopoII inhibitors. Nature 520: 239-242. 2015.

28. Chin KV and Kong AN: Application of DNA microarrays in pharmacogenomics and toxicogenomics. Pharm Res 19: 1773-1778, 2002.

29. Garufi A and D'Orazi G: High glucose dephosphorylates serine 46 and inhibits p53 apoptotic activity. J Exp Clin Cancer Res 33: 79, 2014.

30. Lin J, Shen A, Chen H, Liao J, Xu T, Liu L, Lin J and Peng J: Nitidine chloride inhibits hepatic cancer growth via modulation of multiple signaling pathways. BMC Cancer 14: 729, 2014.

31. Mésange P, Poindessous V, Sabbah M, Escargueil AE, de Gramont $\mathrm{A}$ and Larsen AK: Intrinsic bevacizumab resistance is associated with prolonged activation of autocrine VEGF signaling and hypoxia tolerance in colorectal cancer cells and can be overcome by nintedanib, a small molecule angiokinase inhibitor. Oncotarget 5: 4709-4721, 2014.

32. Baranasic D, Oppermann T, Cheaib M, Cullum J, Schmidt H and Simon M: Genomic characterization of variable surface antigens reveals a telomere position effect as a prerequisite for RNA interference-mediated silencing in paramecium tetraurelia. MBio 5: e01328, 2014.

33. Luo B, Ju S, Muneri CW and Rui R: Effects of histone acetylation status on the early development of in vitro porcine transgenic cloned embryos. Cell Reprogram 17: 41-48. 2015. 